\title{
ANÁLISIS DEL PLAN DE ÁREA DE FILOSOFÍA EN LA INSTITUCIÓN EDUCATIVA SAN VICENTE PARA SU ACTUALIZACIÓN 49
}

\author{
Analysis Of the Plan Area of Philosophy in the \\ School of Saint Vincent for upgrade
}

Daniel Felipe Rodríguez Sánchez*

Rodríguez, D.F. (2016). Análisis del Plan de Área de Filosofía en la Institución Educativa San Vicente para su actualización. Revista PACA, 8, 171-185

\section{Resumen}

En la presente investigación se realizó un análisis de la planeación y del desarrollo curricular de las asignaturas de filosofía en la Institución Educativa San Vicente, ubicada en zona rural de La Plata-Huila. Se acompañó el análisis de una reflexión crítica acerca de las clases limitadas a la transmisión de conocimientos disciplinares, en este caso sobre la historia de la filosofía occidental. Se realizó también un cuestionamiento en relación con la metodología pedagógica tradicional basada en la exposición magistral y el método evaluativo convencional consistente en la elaboración de talleres y evaluaciones escritas que se limitan al pensamiento convergente. De forma coherente y pertinente se propuso un nuevo plan de área de filosofía que trascienda las caracterizadas

\footnotetext{
${ }^{49}$ Título del proyecto investigativo: Evaluación curricular y del Plan de Área de Filosofía de cara a su transformación contextualizada y pertinente, en la Institución Educativa San Vicente de la Plata - Huila. Este se realizó entre agosto de 2015 y enero de 2016.

* Magister en Educación, Filósofo. Docente en la Institución Educativa San Vicente de La Plata, Huila Email: ultimatedfrs1984@gmail.com
} 
prácticas pedagógicas tradicionales. Se partió de la autoevaluación del docente y del punto de vista de los estudiantes mediante una encuesta anónima estructurada y realización de plenarias; finalmente se reestructuró el plan de área de filosofía enfocado a la formación integral, fomentando el criticismo, la reflexión, el análisis racional, la deliberación y la capacidad propositiva en los educandos, mediante procesos estructurados de mediación, negociación, conciliación y concertación.

\section{Abstract}

In the present investigation was carried out an analysis of planning and curriculum development of the subjects of philosophy in the school San Vicente, located in rural area of La Plata-Huila. Analysis of reflection was accompanied criticism classes limited to the transmission of knowledge about discipline, in this case about the history of Western philosophy. There was also a question in relation to the traditional teaching methodology based on the masterful exhibition and the conventional evaluative approach in the preparation of workshops and written evaluations that are limited to the convergent thinking. Of coherent and relevant proposed a new plan of area of philosophy that transcends traditional characterized pedagogical practices. It departed from the self-evaluation of the teacher and from the point of view of students through a structured anonymous survey and preparation of plenary, was finally restructured the philosophy area plan focused on the comprehensive training, encouraging criticism, reflection, rational analysis, deliberation and purposeful capacities in learners, through structured processes of mediation, negotiation conciliation and agreement.

Palabras Claves: Análisis curricular, filosofía, pedagogía y evaluación.

\section{Contextualización}

La formación filosófica en la educación media cobra mayor valor en la medida en que la gran mayoría de la población contemporánea se encuentra enajenada por modas superfluas, programas televisivos banales y el deseo de comprar objetos innecesarios que han generado la ignorancia histórica y política, al igual que la pérdida de valores éticos como la honestidad, el respeto, la responsabilidad y los valores sociales como la fraternidad, la solidaridad, la justicia, la equidad, la tolerancia y la 
laboriosidad, debido a la falta de reflexividad e interés por el bienestar común.

Concederle relevancia a la formación filosófica es necesario sobre todo en el contexto actual de la educación media colombiana que a partir del año 2014 eliminó de las pruebas Saber 11 (antes ICFES) a la filosofía, supuestamente fusionándola con la prueba de lenguaje, lo que nos obliga a preguntarnos: ¿Es posible limitar la formación filosófica a un test de comprensión de lectura? Siendo imposible restringir la formación filosófica y humanista a una prueba formulada como un test de preguntas de selección múltiple con única respuesta sin restarle importancia a este fundamental ámbito del conocimiento humano. Esta situación fue denunciada, atinada y oportunamente por el profesor Germán Vargas Guillén desde la Facultad de Educación de la Universidad Pedagógica (UPN) de Bogotá en su texto "Manifiesto en contra del asesinato de la filosofía en Colombia". En lugar de la exclusión de la Filosofía en la educación media propondría por el contrario fortalecerla incluyéndola obligatoriamente por lo menos desde el grado Noveno, puesto que en la mayoría de establecimientos educativos la confinan hasta los grados Décimo y Undécimo, al igual que las asignaturas de Física y Química, evitando así su desaparición de los planes de estudio como ocurrió en España y Chile.

\section{Cuestiones}

¿Quién promueve que tengamos que deshacernos de la filosofía y de su necesaria práctica en las instituciones educativas, siendo válido el reflexionar sobre el impacto social de la globalización económica debida a la hegemonía del capitalismo imperante en la actualidad, que promueve el ansia por el lucro, la competencia por recursos escasos y el deseo por la constante adquisición de bienes y servicios que ofrece el mercado?

¿Quién promueve que tengamos que deshacernos de la filosofía y de su necesaria práctica en las instituciones educativas estando vigente la globalización política por la generalización de estados fundados formalmente (legalmente) como repúblicas democráticas, pero en la práctica se erigen como regímenes de oligarquía liberal (en términos de Cornelius Castoriadis), puesto que gobierna un grupo selecto de adinerados que se amparan en las leyes para mantener sus privilegios? 
¿Quién promueve que tengamos que deshacernos de la filosofía y de su necesaria práctica en las instituciones educativas estando vigente la globalización cultural evidente en la homogenización de las creencias, prácticas y estilos de vida de las urbes contemporáneas, generando la perdida de cosmovisiones y rituales tradicionales?

¿Por qué este tratamiento a la filosofía, si es fundamental en la formación humanista, para los que deseamos una sociedad democrática, incluyente, participativa, pluralista, respetuosa de la dignidad humana y defensora de los derechos y libertades?

¿Acaso no son precisamente las ciencias humanas y sociales, con el soporte particular de la filosofía, las que tienen la importante misión de trascender la repetición de los planteamientos de autores tradicionales, actualizando sus reflexiones y enfocándose en la búsqueda de propuestas y soluciones alternativas frente a las múltiples problemáticas actuales, orientadas y enfocadas en su objetivo de desarrollar seres reflexivos, autónomos, críticos y propositivos?

\section{Propósito}

Con la presente investigación se propuso realizar un análisis curricular centrado en una revisión del plan de área de la formación filosófica que se imparte en la Institución Educativa San Vicente ubicada en zona rural de La Plata- Huila, con el propósito de revisar los contenidos y las actividades académicas desarrolladas en la enseñanza de esta importante área del conocimiento humano y los procesos de formación integral que ésta facilita y promueve. Esta propuesta se concibió buscando favorecer una formación integral, pertinente y contextualizada, que posibilite el desenvolvimiento y fomento del pensamiento autónomo, crítico y propositivo en los educandos que cursan clases de filosofía en la educación media y, a futuro, en la educación básica secundaria en general, atendiendo a las necesidades e intereses de los educandos y su entorno.

\section{Justificación}

La importancia de un análisis del Plan de Área de Filosofía, de la organización académica de sus asignaturas y de la revisión de las 
metodologías y didácticas hasta ahora utilizadas en los procesos de enseñanza-aprendizaje de la filosofía en la Institución Educativa San Vicente partió de la urgente necesidad de hacer más adecuado, atrayente y agradable para los educandos el aprendizaje de este primordial campo del conocimiento humano y concederle su objetivo fundamental de desarrollar seres reflexivos que se valgan del uso adecuado y ético de su razón.

Lo significativo de realizar una autoevaluación y contextualización de la formación filosófica se debe a la urgente necesidad de brindar una formación humana y académica pertinente, arraigada al contexto históricosocial de los educandos, que sea interesante e importante para la institución educativa y para ellos, por ofrecer conocimientos prácticos que impacten favorablemente sus vidas en lo personal y en su entorno concreto: familia, comunidad, escuela, cultura, barrio, vereda, etc.

Desde esta perspectiva, el análisis del Plan de Área de Filosofía para su actualización y contextualización, es un ejercicio que vale la pena hacer en todas las demás áreas del conocimiento y en otras instituciones educativas tanto públicas como privadas, ya sean rurales o urbanas. El indagar sobre ¿Por qué y para qué caracterizar y actualizar los planes de área de la formación filosófica? es importante debido a la necesidad de rescatar la clase de filosofía actualmente restringida a la instrucción de doctrinas y planteamientos acríticamente, otorgándole su apropiada función de propiciar seres pensantes que reflexionen y deliberen para que impacten positivamente su entorno social.

De esta manera se enfoca la indagación y la reflexión educativa que pretende analizar el plan de estudios de las asignaturas de filosofía en la Institución Educativa San Vicente, investigando temáticas y estrategias que posibiliten en los estudiantes el desarrollo de la reflexión racional, el pensamiento autónomo, el criticismo y la capacidad de proponer alternativas frente a las múltiples problemáticas actuales. Su razón de ser está orientado a solucionar algunos inconvenientes que surgen en el aula durante el transcurso de las clases de filosofía, tales como: la desmotivación de los educandos para abordar el estudio de las ciencias humanas, surgido generalmente de la descontextualización de las temáticas tratadas. 


\section{Marco de Referencia}

Como fundamentación conceptual para el análisis de la formación filosófica en La Institución Educativa San Vicente se analizaron y valoraron algunos aportes de los siguientes autores: Giovanni M. lafrancesco V., Ken Bain, Young Mi Kim, Fernando García Moriyón, Germán Vargas Guillén y Jacques Delors.

Del profesor Giovanni lafrancesco, quien en el contexto colombiano, por medio de su trabajo "La Investigación Pedagógica Formativa", aporta al ámbito de la investigación curricular, didáctica y educativa en general, llamando la atención sobre la importancia de emprender investigaciones orientadas a la actualización de los currículos, el desarrollo de una formación integral y la posibilidad de mejorar la calidad educativa. Incentivando la creación de estrategias, programas, proyectos, prácticas y técnicas para mejorar la calidad educativa, los procesos de formación integral, y las estrategias de enseñanza y aprendizaje. También de este autor el texto "La Gestión y Evaluación Curricular en una Escuela Transformadora", en el que propone un protocolo coherente y pertinente con este trabajo, para estructurar la gestión y evaluación curricular dentro del cual se estructura el Plan de Área de Filosofía propuesto.

De Ken Bain su texto "Lo que hacen los mejores profesores universitarios", en el cual, desde la investigación sobre la excelencia docente en las universidades norteamericanas, resalta la importancia de la autoevaluación de la actividad docente como reflexión constante en aras de mejorar los procesos de enseñanza-aprendizaje, con complemento de la evaluación externa de pares académicos y de los estudiantes para optimizar el proceso educativo.

De Young Mi Kim su texto "Fundamentos y práctica del currículo" realizado para el programa de Educación en Población de la Oficina Regional de Educación para América Latina y el Caribe de la Organización de Naciones Unidas para la Educación, la Ciencia y la Cultura (UNESCO), por su amplia concepción de currículo aproximada a lo que vivimos los educadores en nuestra reflexión pedagógica acerca de las actividades didácticas y evaluativas desarrolladas cotidianamente en el aula. 
De Fernando García Moriyón, quien desde España ha tratado específicamente las múltiples habilidades cognitivas y sociales que debe desarrollar la clase de filosofía en los estudiantes en su texto "¿Para qué sirve enseñar filosofía?".

De Germán Vargas Guillén, quien resalta, defiende y difunde la importancia de la formación filosófica en el contexto actual nacional en su texto "Manifiesto en contra del asesinato de la filosofía en Colombia".

De Jacques Delors, quien en "Los cuatro pilares de la educación" explicita que la educación debe trascender el ámbito del saber disciplinar y desarrollar habilidades prácticas, éticas y sociales.

También se tuvieron en cuenta los lineamientos para la elaboración del currículo de Ciencias Sociales y Filosofía realizado por la alcaldía de Medellín en el año 2014, tanto para la realización del marco conceptual como para la elaboración del Plan de Área de Filosofía propuesto.

\section{Diseño metodológico}

La presente investigación es del tipo de Investigación-Acción-Participativa. Su diseño metodológico se desarrolló conforme con las siguientes cinco fases:

Fase 1: "La participación activa y permanente, a lo largo de todo el proceso de investigación, de la comunidad investigada y que tiene problemas por solucionar".

En este caso, el docente de la asignatura de filosofía que desde su contexto cotidiano en el aula desea mejorar sus prácticas pedagógicas y, de esta manera, optimizar el proceso de aprendizaje de los estudiantes, trabajando en foros y en las clases regulares de filosofía con los educandos, gestores de elementos fundamentales.

Fase 2: "La investigación y el análisis sobre su propia realidad. En ella los sujetos de la investigación son los protagonistas activos de su propio proceso investigativo". 
Lo que se asegura partiendo de la autoevaluación docente de su clase y con la participación voluntaria, directa y explicita de los estudiantes para reelaborar los planes de estudio de filosofía según su contexto social, sus características personales, sus expectativas, intereses y motivaciones personales y de acuerdo con sus problemáticas individuales, sub-grupales y grupales, mediante ágoras, foros y diligenciamiento del instrumento de recolección de información.

Fase 3: "El establecimiento claro de una nueva relación teoría-práctica, entendida esta, como la acción hacia la transformación".

Se realizó durante toda la investigación, no solo mediante la revisión documental teórica, sino construyendo una nueva propuesta de gestión curricular y de plan de estudios para el área de filosofía y para sus asignaturas, tanto en los contenidos de la programación académica, como en las actividades pedagógicas, las estrategias didácticas y la forma de evaluar, de común acuerdo con las propuestas de los educandos.

Fase 4: "La determinación y caracterización de las necesidades de los sectores populares y la creación de una mayor conciencia en relación con la complejidad de las necesidades, la evaluación de los propios recursos y las posibilidades de los grupos de trabajo que asumen la resolución de las primeras (necesidades) y la consecución o adecuación de los segundos (recursos)".

Se logró con el trabajo comprometido de los educandos, desde sus condiciones reales individuales y grupales, de acuerdo con la factibilidad de la investigación y de los recursos de la institución educativa, de los educandos y del educador mediador investigador, sin incurrir en gastos superfluos y sin solicitar a la institución educativa recursos financieros, locativos o de infraestructura, creando espacios dinámicos de formación reflexiva, crítica y deliberante para proponer alternativas frente a los propios desafíos de los educandos, del educador y del entorno real y del contexto concreto en el que se desarrollan las actividades cotidianas.

Fase 5: "Por último, la quinta fase que plantea la organización anticipada de todas las acciones". (lafrancesco, 2013, P.80-81).

Se logró con la elaboración de la propuesta siguiendo el protocolo asumido, 
haciendo seguimiento y evaluación permanente al mismo, tanto por el docente como por los educandos y algunos padres de familia.

\section{Procedimiento}

1. Esta investigación se realizó partiendo de la autoevaluación docente sobre la necesidad de diseñar unas clases de filosofía más atrayentes y llamativas para los estudiantes.

2. Simultáneamente se efectuó una revisión documental de las programaciones de las asignaturas del área de filosofía.

3. Se revisó el Proyecto Educativo Institucional (PEI) en busca del modelo pedagógico institucional (desarrollista cognitivo) para ampliar la metodología pedagógica, usualmente limitada a la clase magistral.

4. También se examinó el Sistema Institucional de Evaluación Escolar (SIEE) que en ningún momento establece una metodología evaluativa coherente con el propósito educativo de formar seres críticos, sino que se limita a establecer la escala evaluativa institucional (de 1 a 5) y la cantidad de áreas establecidas para reprobar el año lectivo.

5. Conjuntamente a la revisión documental se tomó en cuenta la opinión de los estudiantes sobre el desarrollo de la clase mediante encuestas y foros. La encuesta fue aplicada a la totalidad de los estudiantes de grado décimo y undécimo. El objetivo de esta fue conocer su opinión sobre la forma como se imparte la asignatura de filosofía y abrir un espacio para que propongan temáticas de su interés, estrategias pedagógicas, didácticas y evaluativas que tiendan a un mejoramiento de las clases, haciéndolas más atrayentes a los educandos y promoviendo los aprendizajes autónomos, significativos y colaborativos.

6. Se aplicaron las encuestas, se hizo la recolección de información y el tratamiento de los datos, se hicieron las tablas correspondientes, se graficaron los resultados, se hicieron las inferencias pregunta por pregunta, se sacaron las conclusiones y se establecieron las sugerencias correspondientes. 
7. Con la información recolectada a través de la revisión documental tanto de la institución en general analizada en el Proyecto Educativo Institucional, el Sistema Institucional de Evaluación Escolar y la programación académica de filosofía presente en la malla curricular del área de ciencias sociales y, teniendo en cuenta lo mencionado por los estudiantes en la encuesta anónima practicada, se realizó la propuesta de mejoramiento del Área de Filosofía, siguiendo el protocolo propuesto por el profesor Giovanni M. Iafrancesco en su libro "La Gestión y Evaluación Curricular en una Escuela Transformadora".

\section{Población y Muestra}

\section{Población}

La población con la cual se desarrolló esta investigación está representada por los estudiantes de los grados décimo y undécimo de La Institución Educativa San Vicente de La plata-Huila, puesto que son los únicos grados en los que se imparten las asignaturas de filosofía. El grado décimo, que consta de 24 estudiantes ( 12 mujeres y 12 hombres) y el grado undécimo, compuesto de 32 estudiantes ( 18 mujeres y 14 hombres) para una muestra de 56 estudiantes ( 30 mujeres y 26 hombres).

\section{Tipo de muestreo}

Se eligió el "muestreo por grupos o conglomerados", en el cual, la unidad elegida no es un individuo, sino un grupo de personas que se encuentran juntos por causas naturales y que forman un grupo en cuanto son iguales con respecto a las características relacionadas con las variables del estudio. En este tipo de muestreo es esencial que todos los miembros de la población deban figurar en la muestra; es por eso que para el presente estudio, la población es igual a la muestra, así:

\section{Muestra}

Grado 10: 12 hombres y 12 mujeres, en total: 24 educandos. Grado 11: 14 hombres y 18 mujeres, en total: 32 educandos. Total muestra: 26 hombres y 30 mujeres, en total: 56 educandos. 
Todos los educandos que integraron la población y la muestra participaron en la investigación con el consentimiento de sus padres y por voluntad propia, diligenciando una encuesta anónima y participando en plenarias realizadas en la clase de filosofía, las cuales tenían como propósito determinar si existía la necesidad de actualizar los contenidos establecidos en las programaciones académicas del área de filosofía y de sus asignaturas, de revisar los métodos utilizados en la construcción del conocimiento filosófico y verificar si era necesario adecuarlos o transformarlos, de evaluar las estrategias didácticas empleadas en los procesos de enseñanza-aprendizaje en su coherencia y pertinencia y valorar los procesos evaluativos de cara a su mejoramiento.

\section{Resultados}

Como resultado de la presente investigación se resalta que la clase de filosofía no se puede limitar a la trasmisión de conocimientos disciplinares acerca de los principales planteamientos de la historia de la filosofía, sino que, ante todo, debe propiciar la reflexión de múltiples temáticas tradicionales y de actualidad por medio de la comprensión de conceptos fundamentales y el análisis de interrogantes humanos acerca de los valores éticos y morales, los sistemas e ideologías políticas, las tendencias artísticas y los interrogantes cosmológicos, entre otros, para posibilitar en los educandos el desarrollo de su criticismo y capacidad propositiva a través de la argumentación racional y el diálogo asertivo.

Los contenidos de las programaciones académicas propuestas como las metodologías pedagógicas y evaluativas formuladas en el Plan de Área de Filosofía fueron establecidas teniendo en cuenta las opiniones de los educandos plasmadas en las encuestas anónimas diligenciadas voluntariamente por los estudiantes de los grados décimo y undécimo, modificando completamente los temas y el desarrollo de las clases, pasando de unas programaciones basadas en la historia de la filosofía occidental a unas programaciones basadas en problemas filosóficos contextualizados al entorno educativo.

La programación académica de filosofía propuesta incluye temáticas sobre problemáticas sociales actuales como la contaminación ambiental y la depredación de los recursos naturales junto con estrategias para 
mitigarlas. Política actual, historia del conflicto armado en Colombia y el proceso de paz; economía, analizando el impacto de la globalización del capitalismo y, a nivel nacional, se analizarán pros y contras de los tratados de libre comercio, como también el manejo de los presupuestos de la nación y el municipio, entre otros.

Se amplía la metodología pedagógica limitada a la clase magistral, aplicando las TIC en el desarrollo de las clases mediante el uso del Internet en indagaciones de los estudiantes para fundamentar sus exposiciones acerca de temas de su elección. El uso del video beam en algunas presentaciones del docente y los educandos, y para la observación de documentales como "La Educación Prohibida", "La isla de las flores", "Una vida examinada", el análisis de canciones y películas con contenido social. Se establecerá para el desarrollo de la clase la lectura de textos, grupal e individualmente. Se sugiere también la realización de un proyecto investigativo individual para los estudiantes de grado undécimo, y se programarán debates automoderados con temas de actualidad.

Además se proponen diferentes estrategias y tipos evaluativos como la evaluación escrita, la evaluación oral, la elaboración de textos, la autoevaluación, la heteroevaluación, las exposiciones de los estudiantes, la realización y socialización de textos y la participación en clase, por mencionar algunos. Se crearán bancos de preguntas realizadas por los educandos y se organizarán evaluaciones orales por medio de dinámicas interactivas para que los estudiantes lúdicamente se realicen preguntas entre ellos. Las pruebas escritas no se limitarán al pensamiento convergente sino que se realizarán preguntas abiertas y se incluirán interrogantes para que los estudiantes argumenten su opinión sobre un tema visto en clase. Además, se evaluará por medio de exposiciones y la realización de proyectos investigativos con temas elegidos libremente por los estudiantes, fomentando el deseo de profundizar los temas vistos en clase y las inquietudes personales de los educandos, preparándolos para su educación superior.

El alcance de este trabajo se evidencia en la aplicación de un nuevo protocolo de gestión curricular, en la actualización del Plan de Área de Filosofía y de sus asignaturas, dando respuesta a la urgente necesidad de contrarrestar la apatía y el desinterés de los educandos por el estudio de la filosofía y de las ciencias humanas en general, fomentando la superación 
de las dificultades y problemas de la actividad docente con la realización de proyectos de investigación pedagógica, educativa y didáctica, y de actualización curricular y evaluativa como intentos y aproximaciones para abordar los inconvenientes y obstáculos que impiden una educación integral y la formación humana en la Institución Educativa San Vicente, aportando a la resolución de los mismos, sistematizando y difundiendo de forma teórica y práctica, las experiencias exitosas, como resultado de nuestras propias investigaciones pedagógicas formativas.

\section{Referentes Bibliográficos}

- Aristóteles. (1990). Metafisica. Barcelona : Editorial Gredos.

- Bain, K. (2007). Lo que hacen los mejores profesores universitarios. Valencia: Editorial Universitat de Valencia.

- Boche?ski, J. M. (1988). Introducción al pensamiento filosófico. Bogotá: Editorial Herder.

- Castoriadis, C. (2013). La institucion imaginaria de la sociedad. Barcelona: Editores Tusquets.

- Colciencias (1996). Informe de la mision de los sabios "Mision de ciencia,educacion y cultura". Bogotá: Editorial Tercer Mundo.

- Constitucion Politica de Colombia. (1991).

- Delors, J. (1996). Los cuatro pilares de la educacion. Obtenido de Informe a la UNESCO de la Comisión Internacional sobre la Educación para el Siglo XXI: http://uom.uib.cat/digitalAssets/221/221918_9.pdf

- Furtado, J. (1989). La isla de las flores. Obtenido de Youtube: https:// www.youtube.com/watch?v=9fEMHB9kksM

- García Márquez, G. (1994). Por un pais al alcance de los niños. Bogota: Editorial Imprenta Nacional de Colombia.

- García Moriyón, F. (1998). Para qué sirve enseñar filosofía. Obtenido de Universidad Autónoma, Madrid: http://academicos.iems.edu.mx/cired/ docs/ae/pp/fl/aeppflp02pdf01

- lafrancesco, Giovanni M. (2013). La Investigación Pedagógica Formativa: contextos, fundamentos, diseños metodológicos, técnicas, instrumentos y estructuración de proyectos. CORIPET EDITORIAL. Corporación Internacional Pedagogía y Escuela Transformadora CORIPET Ltda. Bogotá, Colombia.

- lafrancesco, Giovanni M. (2013). La gestión y la evaluación curricular en una Escuela Transformadora: Contextos, enfoques, diseños, 
concepto, estructura, modelo holístico, objetivo, planeamiento y protocolo. CORIPET EDITORIAL. Corporación Internacional Pedagogía y Escuela Transformadora CORIPET Ltda. Bogotá, Colombia.

- Kant, E. (1784). Respuesta a la pregunta qué es la ilustracion. Obtenido de http://www.merzbach.de/VoortrekkingUtopia/Datos/texto/ Kant_llustración.pdf

- Lao- Montes, A. (2011). Crisis de la civilizacion occidental capitalista y movimientos antisistémicos. Obtenido de Universidad de Massachusetts: file://C:/Users/PC2/Downloads/635-1367-1-SM.pdf

- Leonard, A. (2009). La historia de las cosas. Obtenido de Youtube: https:/ /www.youtube.com/watch?v=ykfp1WvVqAY

- Lipman, M. (1969). Filosofía para niños. Obtenido de Marco Teórico del Programa de Filosofía para Niños : http://servicios2.abc.gov.ar/ lainstitucion/sistemaeducativo/educacioninicial/capacitacion/ documentoscirculares/2002/filosofiaparaninios.pdf

- López Jiménez, N. E. (2013). "Nuevas tendencias y perspectivas curriculares en educación superior ¿Qué conocimiento, para qué, para quienes?". Bogotá.

- Ministerio de Educacion Nacional. (1994). Ley 115. Obtenido de Ley General de la Educacion: http://www.mineducacion.gov.co/1621/ articles-85906_archivo_pdf.pdf

- Ministerio de Educacion Nacional. (2009). Decreto 1290. Obtenido de Evaluacion del aprendizaje y promocion de los estudiantes de educacion basica y media: http://www.mineducacion.gov.co/1621/articles187765_archivo_pdf_decreto_1290.pdf

- Ministerio de Educacion Nacional. (2010). Documento $N^{\circ} 14$. Obtenido de Orientaciones pedagogicas para la filosofia en la educacion media: h t t p : / / w w w. mined u cacion.gov.co/1621/articles 340033_archivo_pdf_Orientaciones_Pedagogicas_Filosofia_en_Educacion_Media.pdf

- Ministerio de Educación Nacional. (2014). Documento 13. Obtenido de El Plan de Área de Filosofía y de Ciencias Económicas y Políticas: https:/ / w w w. master 2000 . net / recursos/fotos/98/ 13_Filosofia_ciencias_economica_y_politicas.pdf

- Ospina, W. (2012). La lámpara maravillosa. Bogotá: Editorial Mondadori.

- Platon. (1994). Apologia de Socrates. Madrid: Editorial Gredos.

- Platon. (1994). Criton. Madrid: Editorial Gredos.

- Ricouer, P. (2002). Del texto a la accion. Ensayos de hermenéutica II. México: Editorial Fondo de Cultura Economica. 